\title{
Nuclear masses and the number of valence nucleons
}

J. Mendoza-Temis, A. Frank, J. G. Hirsch, J.C. López Vieyra, and I. Morales Instituto de Ciencias Nucleares

Universidad Nacional Autónoma de México, Apdo. Postal 70-543 México 04510 D.F.

\author{
J. Barea
}

Center for Theoretical Physics, Sloane Physics Laboratory

Yale University, P.O. Box 208120 New Haven, Connecticut 06520-8120, USA.

\author{
P. Van Isacker \\ GANIL, BP 55027, F- 14076 Caen Cedex 5, France. \\ V. Velázquez \\ Facultad de Ciencias, Universidad Nacional Autónoma de México, \\ Apartado Postal 70-543, México 04510 D.F.
}

\begin{abstract}
An improved version of the liquid drop model is presented. The addition of two terms, linear and quadratic in the total number of valence nucleons (particles or holes), improves the description of atomic masses, which can be fitted with an r.m.s. error of $1.2 \mathrm{MeV}$. Predictions are analysed an compared those of with established models.

PACS numbers:
\end{abstract}




\section{INTRODUCTION}

An accurate knowledge of nuclear masses is required to understand fundamental processes in nuclear physics. The nuclear mass embodies the net results of all interactions present in the nucleus. The binding energy $B E$ is the difference between the sum of the masses of its constituent free nucleons and the nuclear mass [1].

The study of nuclear mass formulae has a long history since Weizsacker[2], and Bethe and Bacher[3] proposed a formula based on the liquid-drop model, by analogy to a primarily classical system. They considered the nucleus as a very dense, charged liquid drop, where the binding energy is proportional to the volume, i.e. to the mass number $A$, and is reduced by surface and Coulomb effects. Adding the asymmetry term, and the pairing term leads to the familiar form:

$$
B E(N, Z)=a_{v} A-a_{s} A^{2 / 3}-a_{C} \frac{Z(Z-1)}{A^{1 / 3}}-a_{v s y m} \frac{(N-Z)^{2}}{A}+a_{p} \frac{\delta(N, Z)}{\sqrt{A}}
$$

In Eq. (1) the conventional $A^{-1 / 2}$ dependence of the pairing term is adopted [4-7], instead of $A^{-1 / 3}$ form suggested in [8-11]. Numerical fits does not allow to distinguish between them.

In 1966 Myers and Swiatecki which proposed a liquid drop formula including shell and deformation effects [8], which evolved into a macroscopic-microscopic global nuclear mass formula in a collaboration with Moller, Nix and Treiner [9], and later on to the finite range liquid-drop model (FRLDM)[10]. In their macroscopic sector, one contribution was the separation of the asymmetry term in a volumetric and surface part. In 2003, Souza et al.[5] incorporated these surface energy terms in their improved LDM (ILDM). An extra Coulomb term, neglected in most models, was included to take into account corrections to the Coulomb energy associated with the diffuseness of the nuclear surface (proton form-factor correction to the Coulomb energy). The Royer and Gautier mass formula[7] includes a curvature energy and the Wigner cusp, which refers to the extra binding energy present in nuclei with the same number of protons and neutrons.

Danielewicz [6] has shown that, when the surface energy is affected by the particle asymmetry within the system, thermodynamic consistency requires that some of the asymmetry moves to the surface, i.e., an asymmetry skin develops. Minimization of the net nuclear 
energy with respect to the partition of asymmetry produces an expression for the symmetry energy such as in the droplet model[11]. Finally, he shows that the potentially confusing expression for the asymmetry energy is easily comprehended using a capacitor analogy.

Including both surface and Wigner effects, the following extended formula [12] can be written:

$$
\begin{aligned}
B E(N, Z) & =a_{v} A-a_{s} A^{2 / 3}-a_{C} \frac{Z(Z-1)}{A^{1 / 3}}-\frac{a_{v s y m}}{1+\frac{a_{v s y m}}{a_{s s y m}} A^{-1 / 3}} \frac{4 T(T+r)}{A} \\
& +a_{p} \frac{\delta(N, Z)}{\sqrt{A}},
\end{aligned}
$$

where $T \equiv|N-Z| / 2$. The Wigner term has a coefficient modulated by the parameter $r$. It can take values between 0 and 4, which includes the absence of the Wigner term, $r=0$, and two symmetry limits: $r=1$, the exact isospin symmetry associated with the $\operatorname{SU}(2)$ Casimir $T(T+1)$ and $r=4$, the exact spin-isospin symmetry associated with SU(4) Casimir $T(T+4)[12]$.

Nuclear masses and charge radii have been calculated as algebraic functions of the number of valence protons and neutrons [13-15], in a successful approach able to fit more the 2000 known masses with an r.m.s. error smaller than $400 \mathrm{keV}$, employing 28 parameters [15]. In these mass formulas the numbers of valence particles and holes are employed simultaneously, and even in the simplest versions the maximum between two different quantities, associated with spherical and deformed nuclei, is taken [14]. Both facts make it hard to get an intuitive interpretation of the different terms involved. In what follows it will be shown that, by adding only two microscopic terms to the liquid drop model, which are linear and quadratic in the number of valence particles (or holes), it is possible to adjust all known nuclear masses with an r.m.s. of $1.2 \mathrm{MeV}$, a combination of simplicity and predictability which has been found useful in global analysis [12]. Although it is still not competitive with the best mass formulas, it represents a very simple approach, and can serve as a starting point for more sophisticated models [16]. It is worth to mention that Ref. [14] contains a mass formula that is very close to the one employed here, which allows a fit of nuclear masses with similar r.m.s. error. 


\section{MACROSCOPIC MASS FORMULA PLUS SHELL CORRECTIONS}

The main motivation for the present work arose from the striking color-coded pattern observed on the nuclear landscape when plotting the difference between the experimental binding energies[17] and those calculated from Eq. (2) $[12,16,18]$.

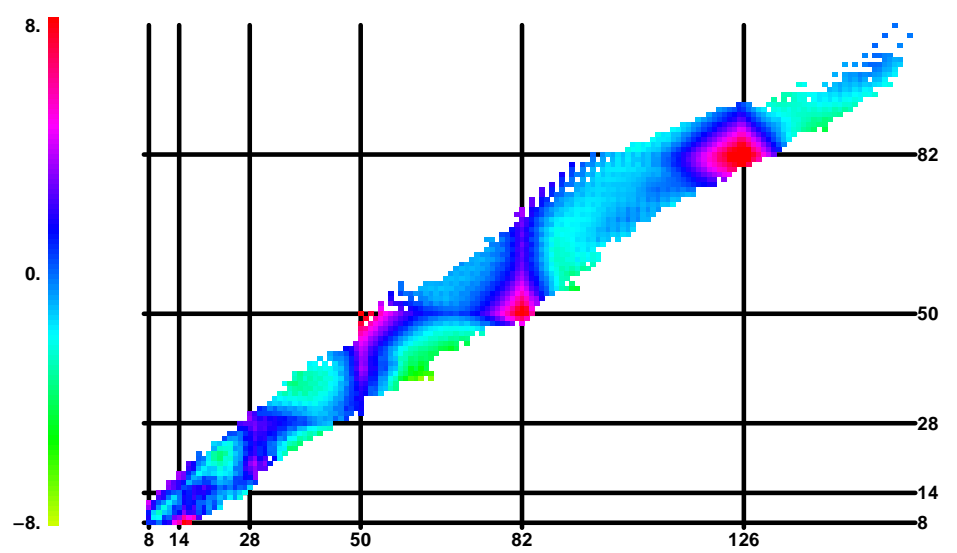

FIG. 1: Residual differences between measured binding energies and those calculated using Eq. 2, with a r.m.s. deviation of $2.40 \mathrm{MeVs}$

The regular pattern displayed in Fig. 1 exhibit the double magic closures as red marks, appearing in those places where the macroscopic mass formula underestimates the binding energies. The biding energy is maximal for those nuclei with $\mathrm{N}$ or $\mathrm{Z}$ equal to $14,28,50,82$ y 126. The pattern suggest to use 14 instead of 20 as a magic number. Around the double closures "diamond like structures" can be observed. They can be parametrized employing $F_{\max }=\left(n_{\nu}+n_{\pi}\right) / 2$ [12], where $n_{\nu}\left(n_{\pi}\right)$ is the number of valence neutron (proton) particles or holes counted from the nearest closed shell. The notation comes from the counting of bosons adopted in the neutron-proton interacting boson model[19]. At mid shells, circular green areas are seen in Fig. 1, where experimental binding energies are smaller than those predicted by Eq. 2.

The number of valence neutrons $n_{\nu}$ is defined by:

$$
\begin{gathered}
n_{\nu}=N-N_{c} \text { if } N \leq N_{\text {med }} \\
n_{\nu}=N_{c+1}-N \text { if } N>N_{\text {med }}
\end{gathered}
$$


where we have introduced the closure magic numbers $N_{c}$ :

$$
N_{c}=8,14,28,50,82,126,184,258, \text { with } c=1,2,3, \ldots, 8
$$

and their midclosures:

$$
N_{\text {med }}=11,21,39,66,104,155,221 .
$$

Similar expressions hold for the number of valence protons $n_{\pi}$. The use of 14 and the exclusion of 20 as a magic number is strongly suggested by the pattern in Fig. 1, and the quality of the fits obtained.

We want to improve the predictive power of Eq. 2 with the inclusion of two extra terms. To keep their parameters as close as possible to its original values, the new terms should have a null average contribution. To this goal their mean is removed by defining

$$
F=\frac{n_{\nu}+n_{\pi}}{2}-\left\langle\frac{n_{\nu}+n_{\pi}}{2}\right\rangle
$$

and

$$
F F=\left(\frac{n_{\nu}+n_{\pi}}{2}\right)^{2}-\left\langle\left(\frac{n_{\nu}+n_{\pi}}{2}\right)^{2}\right\rangle
$$

Introducing the semi-degeneracy $\eta_{c}$ of the shell number $c$ as:

$$
\eta_{c}=\frac{N_{c+1}-N_{c}}{2}
$$

the mean of the valence nucleons can be expressed as:

$$
\begin{gathered}
\left\langle n_{\nu}\right\rangle=\left\langle n_{\pi}\right\rangle=\frac{\eta_{c}}{2} \\
\left\langle\left(\frac{n_{\nu}+n_{\pi}}{2}\right)^{2}\right\rangle=\frac{\left\langle n_{\nu}^{2}\right\rangle+\left\langle n_{\pi}^{2}\right\rangle+2\left\langle n_{\nu}\right\rangle\left\langle n_{\pi}\right\rangle}{4}
\end{gathered}
$$

where

$$
\begin{aligned}
& \left\langle n_{\nu}^{2}\right\rangle=\frac{2 n_{c}^{2}+1}{6} \\
& \left\langle n_{\pi}^{2}\right\rangle=\frac{2 n_{c}^{2}+1}{6}
\end{aligned}
$$

While the removal of their mean values guarantees that the microscopic terms will have no average contribution when all nuclei between closed shells are included, when the analysis 
is restricted to nuclei with measured masses the average value of these two terms is not zero. To compensate for this effect a constant term is added to the complete mass formula, which reads

$$
\begin{aligned}
B E(N, Z) & =a_{v} A-a_{s} A^{2 / 3}-a_{c} \frac{Z(Z-1)}{A^{1 / 3}}-\frac{a_{v s y m}}{1+\frac{a_{v s y m}}{a_{s s y m}} A^{-1 / 3}} \frac{4 T(T+r)}{A} \\
& +a_{p} \frac{\delta(N, Z)}{\sqrt{A}}-a_{f} F+a_{f f} F F+a_{\text {const }}
\end{aligned}
$$

The behavior of the linear term $F$ and the quadratic one $-F F$, after the removal of their average values, are exhibited in Figs. 2a, and 2b.

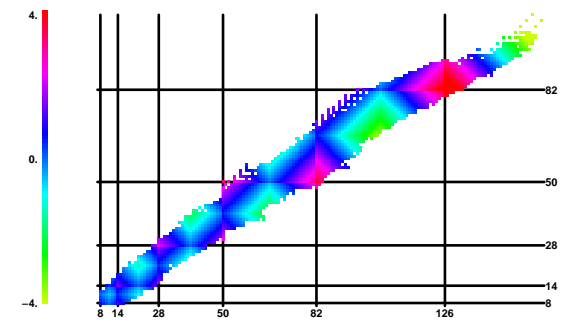

a)

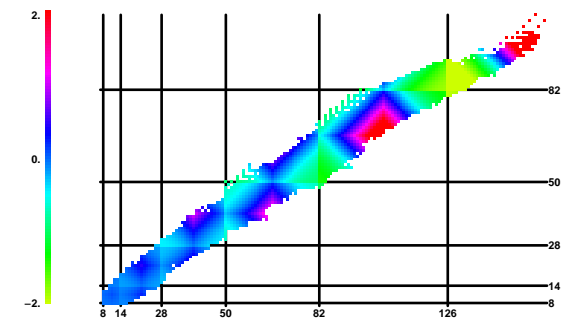

b)

FIG. 2: Behavior of the: a) linear term $F$; b) quadratic term $-F F$ over the entire nuclear landscape along the plane $\mathrm{N}-\mathrm{Z}$.

For $Z<50, N<82$ the linear term dominates over the quadratic, while for heavier nuclei their contributions are comparable. The combined effect $F-F F$ is shown in Fig. 3.

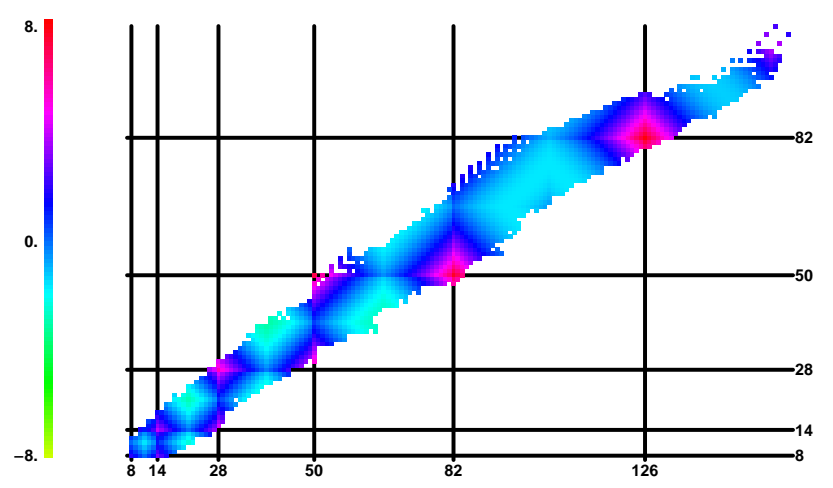

FIG. 3: Shell corrections for a modified macroscopic version of the LDM, due to both terms $F-F F$. 


\section{RESULTS}

In order to get an optimum fit of the coefficients of each formula, we use MINUIT[20], which is conceived as a tool to find the minimum value of a multi-parameter function and analyze the shape of the function around the minimum. The set of data to fit are the masses given in the last Atomic Mass Evaluation[17], which lists atomic and not nuclear masses, the relation between the two being given by

$$
B E_{E X P}(N, Z)=B E_{E X P}^{A M E}(N, Z)+Z\left[B_{e l}(Z=1)\right] m_{e}-B_{e l}(Z)
$$

where $B_{e l}(Z)$ is the total binding energy of the electrons, and its better approximation may be obtained by using[1]

$$
B_{e l}(Z)=1.44381 \times 10^{-5} Z^{2.39}+1.55468 \times 10^{-12} Z^{5.35} \mathrm{MeV}
$$

The parameters in the different mass formulas are adjusted to minimize the r.m.s deviation $\sigma$ defined by

$$
\sigma^{2}=\frac{\sum_{N, Z}\left(B E_{E X P}(N, Z)-B E(N, Z)\right)^{2}}{N_{\text {nucl }}},
$$

which measures the quadratic error between the theoretical binding energies $B E(N, Z)$ and their experimental counterpart $\operatorname{BE} E_{E X P}(N, Z) . N_{\text {nucl }}$ is the total number of nuclei included in the reference set. The parameters obtained after fitting the 2149 nuclei whose masses are reported in the AME03 data set [17] and their respective r.m.s deviation $\sigma$ are listed in Table 1.

The simplest liquid drop mass formula, Eq. (1), allows to fit all known nuclear masses with an average error slightly smaller than 3.0 MeV. Adding surface asymmetry effects improves the fit in $0.25 \mathrm{MeV}$, and the Wigner term in another $0.30 \mathrm{MeV}$.

Finally, its very striking that the inclusion of the two terms proportional to $n_{\pi}+n_{\nu}$ improve the global fit by $1.1 \mathrm{MeV}$, to a final r.m.s. error of $1.3 \mathrm{MeV}$.

While the coefficients of the volume, surface, Coulomb and pairing terms are fairly constant, the asymmetry term is strongly affected by the presence of the surface and the Wigner corrections, which means that these terms are strongly correlated. The constant $a_{\text {cont }}=-$ $0.2759 \mathrm{MeV}$ simply corrects for the average contribution of the microscopic terms for all nuclei with known masses. 


\begin{tabular}{|c|c|c|c|c|}
\hline COEFFICIENT & Eq. (1) & Eq. (2) & Eq. (2) & Eq. (14) \\
\hline$a_{v}$ & 15.671 & 15.714 & 15.454 & 15.454 \\
\hline$a_{s}$ & 17.701 & 18.078 & 17.053 & 17.053 \\
\hline$a_{c}$ & 0.7120 & 0.7070 & 0.6891 & 0.6891 \\
\hline$a_{v s y m}$ & 23.077 & 27.206 & 44.507 & 44.507 \\
\hline$a_{\text {ssym }}$ & - & 25.145 & 6.9071 & 6.9071 \\
\hline$a_{p}$ & 12.735 & 12.797 & 12.444 & 12.444 \\
\hline$a_{\text {const }}$ & - & - & - & -0.2759 \\
\hline$a_{f}$ & - & - & - & 1.3349 \\
\hline$a_{f f}$ & - & - & - & 0.0469 \\
\hline$r$ & - & 0 & 2.2437 & 2.2437 \\
\hline$\sigma$ & 2.9408 & 2.6921 & 2.4007 & 1.3317 \\
\hline mean & -0.0722 & -0.0359 & -0.0223 & -0.0016 \\
\hline
\end{tabular}

TABLE I: Coefficients [in $\mathrm{MeV}$ ] for the different mass formulas

If the curvature term $a_{k} A^{1 / 3}$ and the Coulomb diffuseness correction $c_{d} \frac{Z^{2}}{A}$ are included, the global fit can be improved to $1.2 \mathrm{MeV}[18]$.

Fig. 4 displays the color-coded pattern of the residual differences between experimental binding energies[17] and those calculated by using Eq. (14), which includes shell closure effects. Notice that the scale runs between -2.0 and $2.0 \mathrm{MeV}$, amplifying small differences.

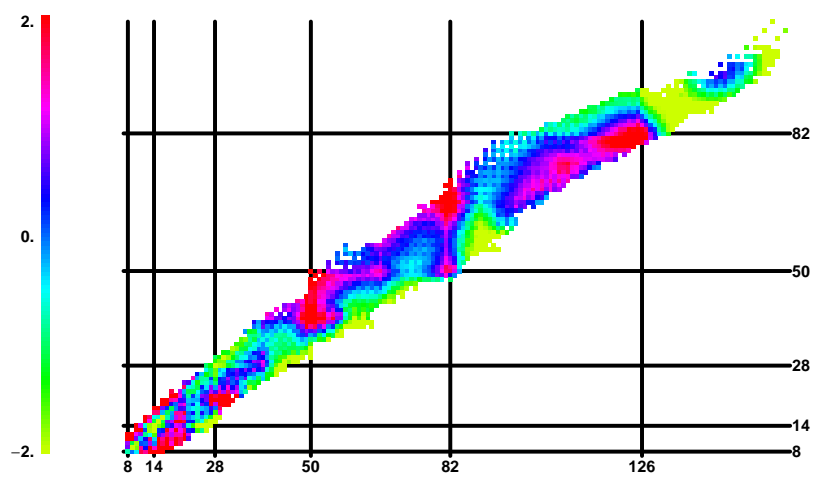

FIG. 4: Residual differences on the $\mathrm{N}-\mathrm{Z}$ plane, between measured binding energies and those calculated using Eq. (14).

It is worth to mention that we have studied a generalized version of Eq. (14), including 
microscopic contributions of the form $a_{1 \pi} n_{\pi}+a_{1 \nu} n_{\nu}+a_{2 \pi} n_{\pi}^{2}+a_{\pi \nu} n_{\pi} n_{\nu}+a_{2 \nu} n_{\nu}^{2}$. The numerical results show that the best parameters satisfy $a_{1 \pi} \approx a_{1 \nu}$ and $a_{2 \pi} \approx a_{2 \nu} \approx a_{\pi \nu} / 2$. It provides a strong empirical support for the use of $n_{\pi}+n_{\nu}$ as the microscopic variable. They can interpreted the as isoscalar global monopole corrections to the binding energies which can be extracted from a microscopic Hamiltonian [21].

\section{TESTS AND PREDICTIONS}

\section{A. AME95-03 test}

In order to check the reliability of the nuclear mass formulas, we use the AME95 - AME03 test employed in Ref. [1]. It consists in selecting only the 1760 nuclei whose masses are already included in the AME95 compilation[22], and predict the 389 nuclear masses which are present in AME03[17] but not in AME95[22]. It should be stressed that, for the sake of consistency, we always employ the AME03 data set, and use AME95 only to select the restricted set of nuclei to be fitted.

The results of the reliability test applied to the different mass models are displayed in Table II.

\begin{tabular}{|c|c|c|}
\hline FORMULA & \multicolumn{2}{|c|}{$\begin{array}{c}\text { AME95-AME03 TEST (r.m.s. in MeVs) } \\
\text { FITTED }\end{array}$} \\
\hline Eq. (1) & 2.7932 & PREDICTED \\
\hline Eq. (2) & 2.4980 & 2.2148 \\
\hline Eq. (14) & 1.3681 & 1.3185 \\
\hline Eq. (14) extended & 1.2390 & 1.0751 \\
\hline
\end{tabular}

TABLE II: Results for the Reliability tests

As seen in Table II, all mass formulas are quite stable in their predictions, having an r.m.S. deviation for the predicted nuclei smaller than the fitting error. The microscopic formulas, Eq. (14) and its extension, have a deviation close to $1 \mathrm{MeV}$ in the prediction. 


\section{B. Predictions up to the drip lines}

Drip lines are the boundary beyond which neutron(proton)-rich nuclei are unstable against neutron(proton) emission. In other words, the drip lines are the lines on the $\mathrm{N}-\mathrm{Z}$ plane where the neutron(proton) separation energy is zero. Thus, an unstable atomic nucleus beyond the drip lines will leak free nucleons. In astrophysics, the neutron drip line is important in discussions of nucleosynthesis and neutron stars.

Having showed that Eq. (14) successfully satisfies the AME95-03 test, it is relevant to investigate its predictions up to the drip lines. Although binding energies are predicted with a precision better than $1 \%$ by the simplest liquid drop mass formula, Eq. (1), its error is still an order of magnitude too large for precise nucleosynthesis studies. One useful way to find regions of stability is to substract to the mass predictions its own macroscopic part. The microscopic differences will exhibit in an enhanced way the regions where binding energies in a certain model are larger than its macroscopic estimation. It should be stressed that, due to the strong differences in the asymmetry coefficients in the different mass formulas, there is not such a thing as a "universal liquid drop model" to be substracted from all mass predictions. The proper macroscopic prediction must be substracted in each model, which can be obtained by the best fit of the LDM, Eq (1), of the theoretical binding energies associated with each model.

In the case of Eq. (14), the microscopic prediction is simply included in the two terms dependent on valence occupation numbers. They are shown in the left-hand side of Figs. 5 and 6. As expected from the discussion in the previous sections, the new stability regions for superheavy nuclei predicted by the model are associated with the shell closures and midclo-

sures. In particular, the "diamond like" stability pattern is predicted to exist around ${ }^{184} \mathrm{~Pb}^{266}$, ${ }^{155} 104^{259},{ }^{184} 126^{310}$ and ${ }^{221} 104^{335}$. Some of the are heavy double-magic nuclei, while some others are mid-shell nuclei predicted as stable due to the presence of quadratic term.

For comparison we have selected two of the most successful macroscopic-microscopic models: the Finite Range Droplet Model (FRDM) [10] and Duflo-Zuker (DZ) model [15]. For the FRDM the separation of macroscopic and microscopic contributions is performed by the authors. The microscopic contribution is plotted in the right-hand side of Fig. 5. It is 
remarkable that ${ }^{184} \mathrm{~Pb}^{266}$ is already predicted to be particularly stable, and the midshell nuclei ${ }^{155} 104^{259}$ and ${ }^{221} 104^{335}$ are also exhibited as relatively stable. However, the region of stable nuclei around ${ }^{184} 126^{310}$ is fairly spread.

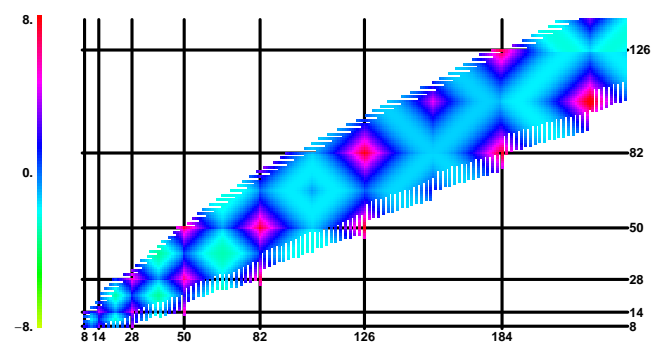

a)

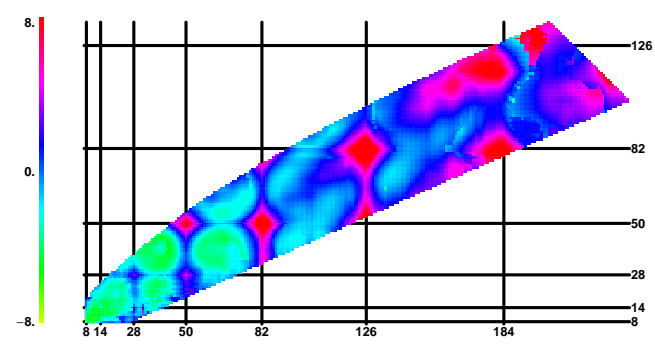

b)

FIG. 5: Predictions up to the drip lines using our formula which includes shell corrections compared with the FRDLM of Moller and Nix

In the DZ model with 28 parameters there is a macroscopic sector, but it is referred as a base line, not a proper liquid drop, in Ref. $[13,14]$. The microscopic corrections shown in Fig. 6 were obtained removing a liquid drop calculation fitted to the DZ predictions. The stability around ${ }^{184} \mathrm{~Pb}^{266}$ is already there, but instead of ${ }^{155} 104^{259}$ the stability region is displaced to a heavier region by about ten nucleons, both for protons and neutrons.

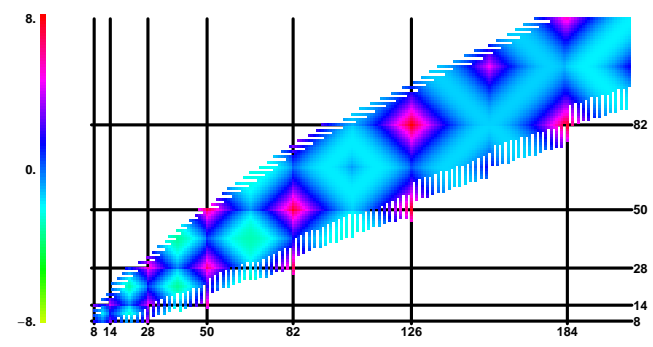

a)

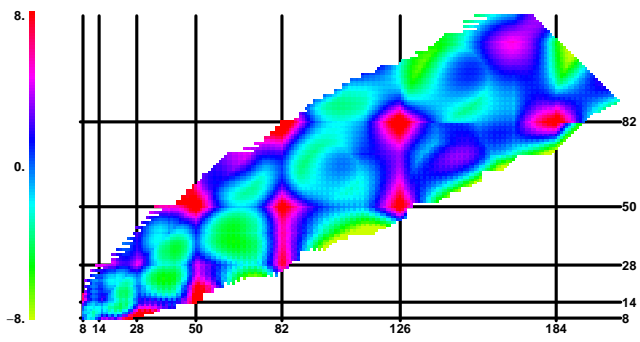

b)

FIG. 6: Predictions up to the drip lines using our formula which includes shell corrections compared with the Duflo and Zuker mass formula

As a further test of the present model, cubic terms in $n_{\pi}+n_{\nu}$ were included in a global fit. The r.m.s. error did not exhibit any improvement. However, this cubic term has a negative coefficient, which makes the quadratic contribution smoother, as shown in Fig. 7

The double closure at magic numbers and mid-shell closures seems to be robust predictions of the present model. It would be expected that refined versions of this model, with r.m.s. 


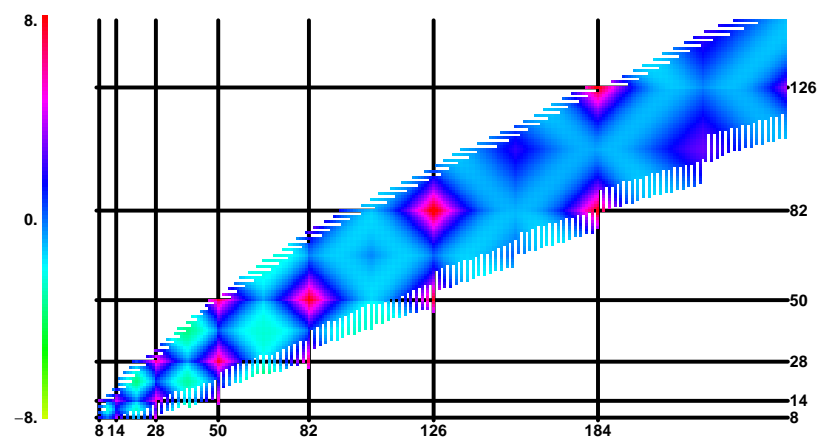

FIG. 7: Predictions up to the drip lines using our formula which includes shell corrections up to cubic terms

fitting capabilities closer to the FRDM and the DZ models, would have slight modifications, but the islands of stability would remain where there are predicted now.

\section{FINAL REMARKS}

The study of the differences between experimental binding energies and those calculated with macroscopic mass formulas, plotted on the plane $\mathrm{N}-\mathrm{Z}$ over the entire nuclear landscape, motivated the introduction of a simple parametrization which includes shell corrections by using terms like $\left(n_{\nu}+n_{\pi}\right) / 2$ to fit the nuclear landscape with a r.m.s. deviation of $1.3 \mathrm{MeV}$.

In order to retain the validity of the parameters fitted using the macroscopic expression, Eq.(2), the average contributions of the microscopic terms were removed. Proceeding in this way, we have obtained a r.m.s. deviation of $1.3 \mathrm{MeV}$ starting from a formula that takes into account the surface asymmetry and the Wigner term. When the curvature and the correction to the Coulomb energy were added the fits improved by $100 \mathrm{keV}[18]$.

The AME95 - AME03 test presented in the report of Lunney et al.[1] was applied successfully to the model predictions. Islands of stability were predicted for superheavy nuclei with magic or mid-shell proton and neutron numbers. They were not far from the predictions of more sophisticated models like the FRDM and DZ models. Having included the most relevant shell corrections, the present model could serve as a basis for more elaborated techniques in the quest for precise nuclear mass predictions. 
This work was supported in part by Conacyt, México and DGAPA, UNAM.

[1] D. Lunney, J.M. Pearson, Rev. Mod. Phys. 75 (2003) 1021.

[2] C.F. von Weizscker, Z Phys. C 96 (1935) 431.

[3] H.A. Bethe, R.F. Bacher, Rev. Mod. Phys. 8 (1936) 82.

[4] A. Bohr, B.R. Mottelson, "Nuclear Structure - Vol. I", World Scientific (1988).

[5] S. R. Souza, P. Danielewicz, S. Das Gupta, R. Donangelo, W. A. Friedman, W. G. Lynch, W. P. Tan, Phys. Rev. C 67 (2003) 051602.

[6] P. Danielewicz, Nucl. Phys. A 727 (2003) 233-268.

[7] G. Royer and C. Gautier, Phys. Rev. C 73 (2006) 067302.

[8] W.D. Myers and W.J. Swiatecki, Nucl. Phys. 81 (1966) 1.

[9] P. Moller, W.D. Myers, W.J. Swiatecki and J. Treiner, Atomic Data Nucl. Data Tables 39 (1988) 225.

[10] P. Moller, J.R. Nix, W.D. Myers, and W.J. Swiatecki, At. Data Nucl. Data Tables 59 (1995) 185.

[11] W.D. Myers, Droplet Model of Atomic Nuclei, IFI/Plenum, New York (1977).

[12] P. Van Isacker, 12th Int. Symp. On Capt. Gamma-Ray Spectr. and Related Topics. AIP Conf. Proc. Vol. 819 (2006) 57-64 ; A. E. L. Dieperink and P. Van Isacker, Eur. Phys. J. A 32 (2007) 11.

[13] J. Duflo, Nucl. Phys. A576 (1994) 29.

[14] A.P. Zuker, Nucl. Phys. A576 (1994) 65.

[15] J. Duflo and A. P. Zuker, Phys. Rev. C 52 (1995) R23.

[16] A. Frank, J. C. López Vieyra, J. Barea, J. G. Hirsch, V. Velázquez and P. Van Isacker, AIP Conf. Proc. Vol. 819 (2006) 151; I. Morales, A. Frank, J. C. López-Vieyra, J. Barea, J. G. Hirsch, V. Velázquez and P. Van Isacker, Int. Journal of Mod. Phys. E 15 (2006) 1855; A. Frank, J. C. López-Vieyra, I. Morales, J. Barea, J.G. Hirsch, V. Velázquez and P. Van Isacker, Rev. Mex. Fis. S 52 (2006) 17; A. Frank, J. C. López Vieyra, J. Barea, J. G. Hirsch, V. Velázquez and P. Van Isacker, AIP Conf. Proc. Vol. 884 (2007) 61.

[17] G. Audi, A. H. Wapstra, and Thibault, Nucl. Phys. A 729 (2003) 337.

[18] Joel Mendoza-Temis, Master Thesis in Physics, ICN-UNAM (2007).

[19] A. Arima, T. Otsuka, F. lachello, and I. Talmi, Phys. Lett. B 66 (1977) 205. 
[20] F. James, Minuit: Function Minimization and Error Analysis Reference Manual, Version 94.1, CERN (1994).

[21] E. Caurier, et al., Phys. Rev. C 359 (1999) 2033.

[22] G. Audi, A. H. Wapstra, and Thibault, Nucl. Phys. A 595, (1995) 409. 